\title{
Design and Performance of a Miniaturized, Low-Energy, Large Beam Spot Electron Flood Gun
}

\author{
Chenhui Deng ${ }^{1,2, *}$, Li Han ${ }^{1,2}$ and Yan Wang ${ }^{1}$ \\ 1 Institute of Electrical Engineering, Chinese Academy of Sciences, Beijing 100190, China; \\ lihan@mail.iee.ac.cn (L.H.); wangysnow@mail.iee.ac.cn (Y.W.) \\ 2 School of Electronic, Electrical and Communication Engineering, University of Chinese Academy of Sciences, \\ Beijing 100049, China \\ * Correspondence: dengch@mail.iee.ac.cn
}

Citation: Deng, C.; Han, L.; Wang, Y. Design and Performance of a Miniaturized, Low-Energy, Large Beam Spot Electron Flood Gun. Electronics 2021, 10, 648. https:// doi.org/10.3390/electronics10060648

Academic Editor: Changhwan Shin

Received: 31 January 2021

Accepted: 3 March 2021

Published: 11 March 2021

Publisher's Note: MDPI stays neutral with regard to jurisdictional claims in published maps and institutional affiliations.

Copyright: (c) 2021 by the authors. Licensee MDPI, Basel, Switzerland. This article is an open access article distributed under the terms and conditions of the Creative Commons Attribution (CC BY) license (https:// creativecommons.org/licenses/by/ $4.0 /)$.

\begin{abstract}
Charge accumulation often occurs in electron optics equipment and interferes with their operation. The trouble can be handled by using the electron flood gun. However, there are still some scenarios where neutralization is not as desired. To achieve a better charge neutralization effect and to facilitate work in confined spaces, a small size, low-energy electron flood gun providing a large area and uniform electron beam has been required. This article employs Munro's Electron Beam Software (MEBS) to simulate the effect of the structure parameters on the performance of the beam. Based on the simulation results, the electron flood gun is processed and assembled. To verify the performance of the electron flood gun, this paper proposes a new "pinhole scanning method". By using the method, we have achieved in-situ measurements of beam current and beam spot. The experimental results generally match the simulation results, which demonstrates that the electron flood gun has good performance and is likely to have many applications.
\end{abstract}

Keywords: charge phenomena; electron flood gun; numerical simulation; pinhole scanning method

\section{Introduction}

When charged particles irradiate poor electrical conductivity materials such as insulators and semiconductors, the accumulation of charge is generated on the materials, this scenario is known as the charge phenomena [1-4]. The phenomena will form an electric field on the surface of the sample, which will then change the trajectory of incident electrons and ejected electrons, causing a serious impact on the use of electron optics equipment. This can be seen in the use of electron scanning microscopes [5,6] (SEM), where the image resolution is reduced due to charge phenomena, making it difficult to determine the topography of the surface. In addition, the charge phenomena affect other modes of operations such as EBSD, EDS, and so on.

In addition, charge phenomena can also affect surface composition analysis equipment such as X-ray photoelectron spectroscopy [7-9] (XPS), Auger electron spectroscopy [10] (AES), secondary ion mass spectrometry [11,12] (SIMS), and so on. The phenomena alter the potential of the sample surface, making it difficult to accurately determine the binding energy to identify the element. In electron-beam processing equipment such as electron-beam lithography [13], resist discharge and line distortion can occur. During ion implantation [14], the charge phenomena affect the effectiveness of ion implantation.

The charge phenomena are thought to result from an unequal number of incident electrons and emitted electrons [1-4]. Several methods have been developed to minimize the effect caused by the charge phenomena, such as reducing the resistivity of the sample $[15,16]$, varying the environmental pressure $[13,17]$, and neutralizing the residual charge on the sample [7-12]. Electron neutralization has significant advantages over other methods, mainly in the precise control of the amount of charge injected to achieve a good neutralization effect, while maintaining the original experimental conditions and not 
causing sample contamination and damage. Therefore, this method is used in XPS [7-9], AES [10], and SIMS [11,12].

Using the electron flood gun is a common way in charge neutralization [7-12]. The neutralization effect depends on the performance of the electron flood gun; special attention should be paid to possible insufficient neutralization, over-neutralization $[8,10]$, and damage to the sample [12]. The basic requirements are as follows. Low-energy is necessary to avoid negative charge build-up and sample damage. The beam spot should be as large as possible according to the application conditions in order to improve neutralization efficiency. Considering that flood guns are mostly used for integration instead of used alone, they are required to work in confined environments, so miniaturization is important to them. Based on these considerations, our research group decided to design a low-energy, large beam spot, miniaturized electron flood gun.

Our work on the electron flood gun will be presented by both design and performance in this article. In the design aspects, the traditional three-electrodes structure of a cathode, an anode, and a gird was used. Further numerical simulations were used to determine the size of the three electrodes and the distance between them. Based on the simulation results, the electron flood gun has been manufactured and assembled. To validate the performance of the electron flood, a new method-the "pinhole scanning method"-was proposed by our group. Based on this, a test system was established and in-situ measurements of the electron beam current and the beam spot were achieved. Through the comparison between simulation and experimental results, our design is believed to have good performance; it meets the design requirements of miniaturization, low energy, and a large beam spot.

\section{Design Aspects}

Considering the design requirements of miniaturization, low-energy, and large spot, the structure of the electron flood gun should be as simple as possible. As a result, the focusing elements have been omitted in the design, and the three-electrodes structure composed of a cathode, a grid, and an anode has been adopted. In view of that, the structures of low-energy electron guns are mostly rotational-axis symmetry [18,19], the electron gun designed by our research group follows this structure, as shown in Figure 1, where the r-direction is the radial direction and the $\mathrm{z}$-direction is the axial direction, the point $\mathrm{O}$ is the center of the cathode. The distance from the cathode and the grid and the distance from the grid to the anode are represented by $d_{c g}$ and $d_{g a}$, respectively.

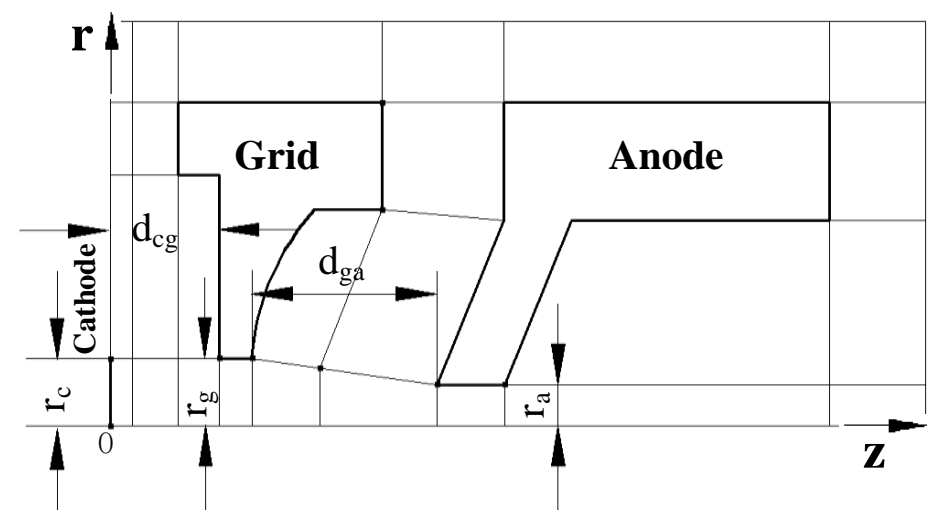

Figure 1. Design outline of the electron flood gun.

The specific parameters of each electrode in the electron gun have been determined based on the calculation results of Munro's Electron Beam Software (MEBS) which is used worldwide by numerous electron microscope manufacturers and by manufacturers of charged particle optical equipment for the semiconductor industry. The MEBS was used to determine the geometric parameters and potentials of each electrode.

Taking into account the performance requirements for large beam spots and based on simulation results, a disk cathode with a diameter of $1 \mathrm{~mm}$ was applied in the electron gun. 
Important structural parameters such as the size of the grid, the size of anode, the distance between grid and cathode, and the distance between grid and anode are determined with reference to the simulation results calculated by the MEBS. In addition, it is necessary to ensure the insulation between each electrode during the design. After determining the approximate value of each important parameter, considering the effect of space charge, the electron beam trajectory (red line) is shown in Figure 2, and the green line represents the electric field distribution.

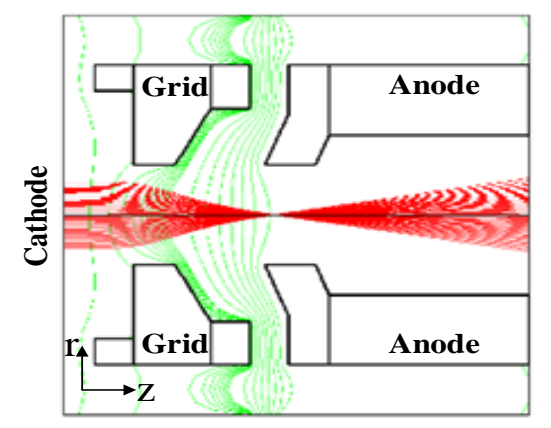

Figure 2. Electron trajectory calculated by MEBS.

The electron flood gun is designed as the detachable structure to the analysis of the influence of structural parameters on the performance of the electron beam and to facilitate adjusting the performance of the electron beam. The detachable structure is mainly composed of a cathode, a grid, an anode, and insulating ceramics, as demonstrated in Figure 3a. The cathode is a $\mathrm{LaB}_{6}$ disk emitter which gives a high emission current at low temperatures compared to tungsten filaments and a lifetime of over $500 \mathrm{~h}$. From Figure 3b, it can be seen that the assembled electron flood gun body is approximately $2 \mathrm{~cm}$ long, which has the superiority of small size.
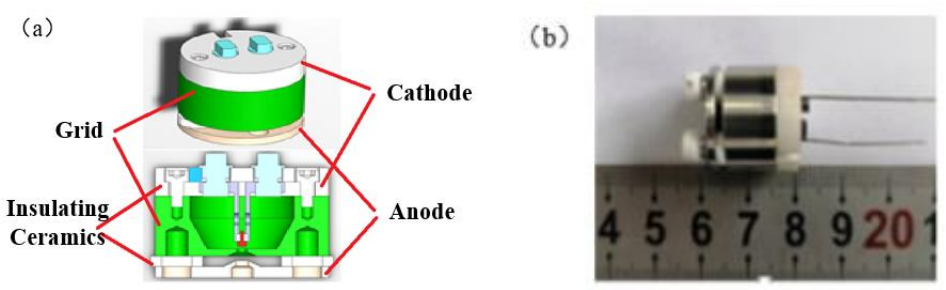

Figure 3. (a) Schematic diagram of the electron flood gun; (b) picture of the electron flood gun.

The detachable structure makes installation extremely important. The detailed installation procedures are based on the cathode. After the position of the cathode is determined, the position of the grid and anode is determined successively according to it. The accuracy of the position of the cathode center is a significant parameter. If the elements of the electron gun are poorly aligned, it will result in an asymmetrical profile of the electron beam and even astigmatism. When the grid voltage is adjusted, the electron beam will change irregularly, instead of being concentrically larger or smaller which may cause an extremely inconvenient operation or even serious damages to the samples in the process of neutralization. In addition to the removable structure for easy adjustment to facilitate center alignment, four additional adjustment screws are designed to ensure the accuracy of the center alignment.

\section{Method}

After the design and assembly, evaluating the performance of the electron gun according to the design requirements is our main task. Miniaturization is directly reflected in the appearance, as shown in Figure $3 \mathrm{~b}$. While other design requirements need to be verified by measuring the beam current and beam spot of the electron beam. 
In order to measure the beam current, our group adopted a maneuverable, efficient, and common method-the Faraday cup method [20].

There are some difficulties in measuring beam spots compared to beam currents. CCD observation method [21] and the occlusion scan method [22] are usually used for beam spot measurement. The CCD observation method depends on the fluorescence generated by the electrons irradiated on the fluorescence screen. This method has some difficulties in quantitatively calculating the beam spot size and is limited by the range of incident electrons energy, especially when the incident energy is low and the fluorescence cannot be excited. The principle of the occlusion scan method is to analyze the electrons received in the Faraday cup. Nevertheless, on account of the interaction between the electron beam and the solid, an interference signal mainly composed of secondary electrons is generated in this process, which greatly affects the accuracy during the measurement.

A new method called the "pinhole scanning method" is proposed to solve the above problem. The method is also intended for in-situ measurement of beam current and beam spot, an illustration is shown in Figure 4. Two Faraday cups are fixed side by side on the stage, the one with a larger hole is termed "holey Faraday cup", the other with a pinhole is named "apertured Faraday cup". The holey Faraday cup is used to measure the current of the beam, while the apertured Faraday cup is applied for measuring the diameter and uniformity of the beam spot. Owing to the grounded metal shield fixed above the apertured Faraday cup, the method is free of the influence of secondary electrons.

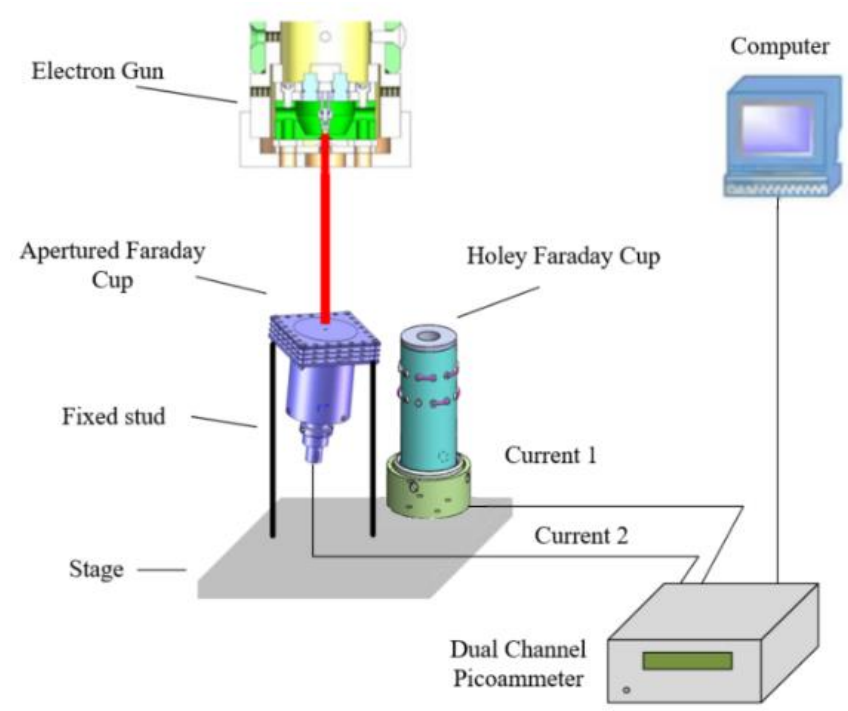

Figure 4. Schematic diagram of the pinhole scanning method.

According to the proposed principle of the pinhole scanning method, the electron beam performance test system has been built up. The system is mainly composed of a vacuum subsystem, electron gun, and its control subsystem, stage subsystem, and data acquisition subsystem which includes "apertured Faraday cup", "holey Faraday cup", dual-channel picoammeter (model 6482 by Keithley Instruments Company), and computer as shown in Figure 4.

The steps for using the pinhole scanning method are described in detail below. Initially, move the "holey Faraday cup" under the electron gun and ensure that the electrons emitted from the gun fully enter it. Meanwhile, the beam current is measured by a dual-channel picoammeter. Then, the "apertured Faraday cup" is placed under the electron gun for receiving electrons. What calls for special attention is that the diameter of the aperture must be smaller than the diameter of the beam spot. Next, the aperture will move with the stage at a constant speed to achieve a relative movement of the beam spot. The trajectory of the aperture is indicated in Figure 5, where the continuous dash line represents the path of the aperture. It is necessary to guarantee the moving range of the aperture completely covers 
the beam spot area. During the moving process, the electrons received by the apertured Faraday cup flow into model 6482, the current measured by the instrument are displayed in the computer. The diameter and uniformity of the beam spot are acquired through further comparison and analysis. The method realizes the measurement of beam current and beam spot under in situ conditions. The following section will provide specific results.

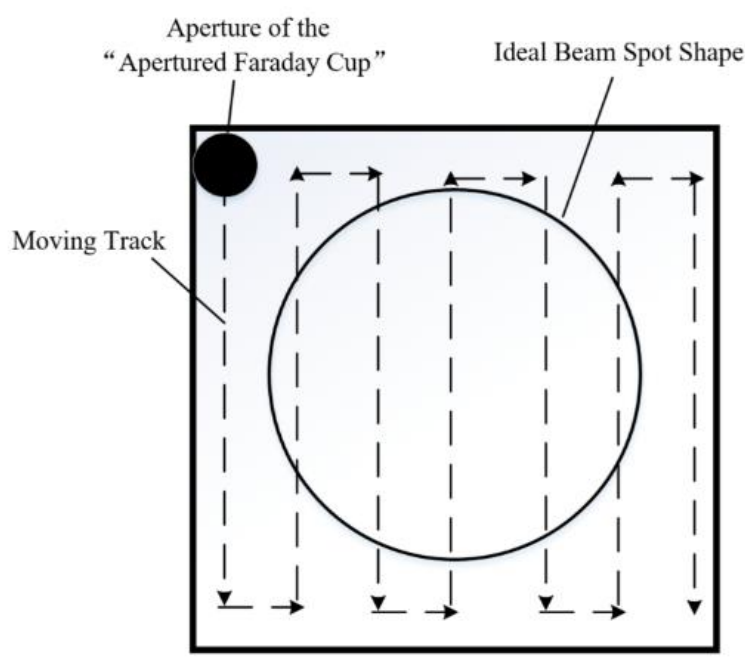

Figure 5. Diagram of the movement of the aperture.

\section{Results}

The electron beam performance experimental results are presented below. The relationship between the beam current and beam energy obtained by the holey Faraday cup is demonstrated in Figure 6; the beam current is proportional to the beam energy under the condition that the grid voltage and the cathode current have been kept constant.

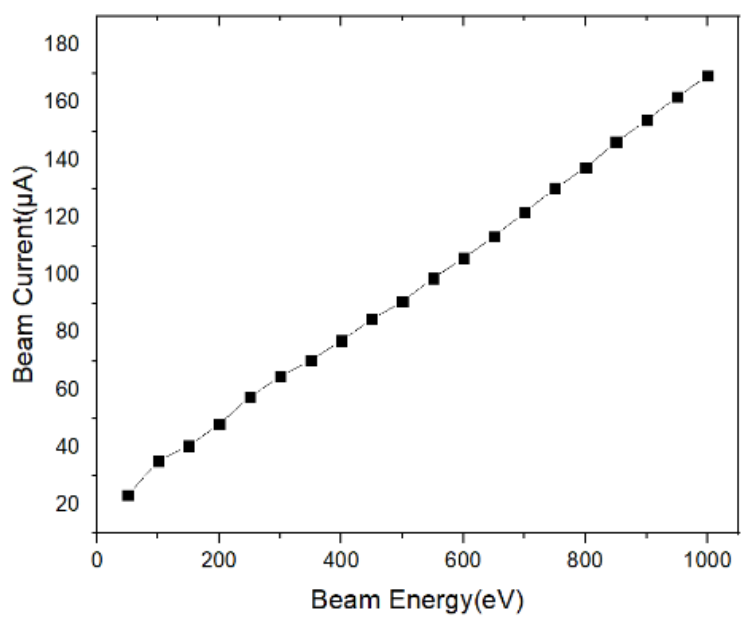

Figure 6. Beam current varies as beam energy changes. (grid voltage $=10 \mathrm{~V}$, cathode current $=1.7 \mathrm{~A}$ ).

Since the structure we adopted is composed of a cathode, a grid, and an anode, the impact of the grid voltage on the performance of the electron beam, especially on the beam current and beam spot, constitutes the main factor to be concerned about. Simulation and experimental results of the beam current basically matched well as demonstrated in Figure 7. It can be inferred that the electron beam is completely suppressed with the grid voltage less than $-6 \mathrm{~V}$. In addition, given the designed maximum value of the beam current $(200 \mu \mathrm{A})$, the voltage of the grid is recommended below $10 \mathrm{~V}$. Within a certain range of $-6 \mathrm{~V}$ to $10 \mathrm{~V}$, the beam current is continuously increasing when the grid voltage augments. 


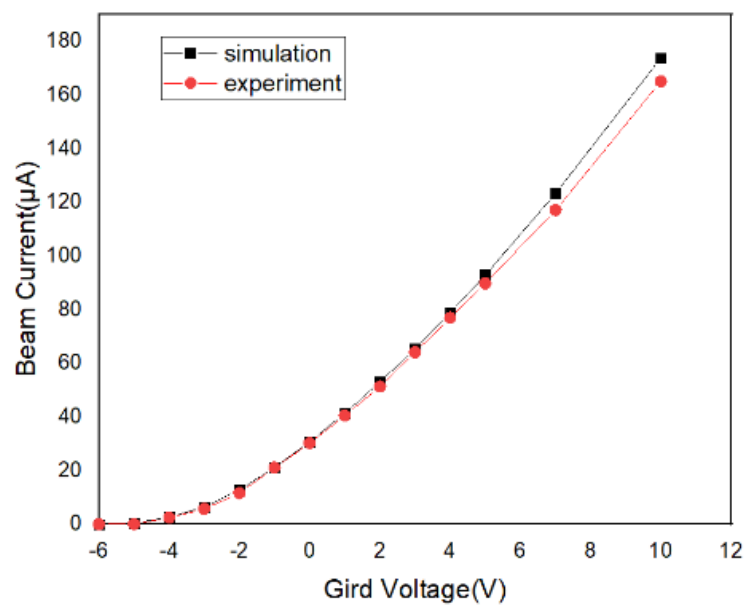

Figure 7. Effect of the grid voltage on the electron beam current. (beam energy $=1000 \mathrm{eV}$, cathode current $=1.7 \mathrm{~A})$.

After measuring the electron beam current by the holey Faraday cup, the beam spot diameter can be measured by using the apertured Faraday cup under in-situ conditions. Taking the working distance of $10 \mathrm{~mm}$ as an example, the simulation and experimental results are slightly different as shown in Figure 8. When the grid voltage is negative, the experimental result is higher than the simulation value. However, when the voltage turns positive, the opposite tendency appears: the experimental result becomes lower than the simulation value. Specifically, compared with the data in the simulation, whether the grid voltage is negative or positive, the corresponding desired effect is not achieved. The reason for the phenomena is probably that compared with the circumstances in the simulation, the insufficient electric field during experiments leads to the inefficiency of the grid control capability.

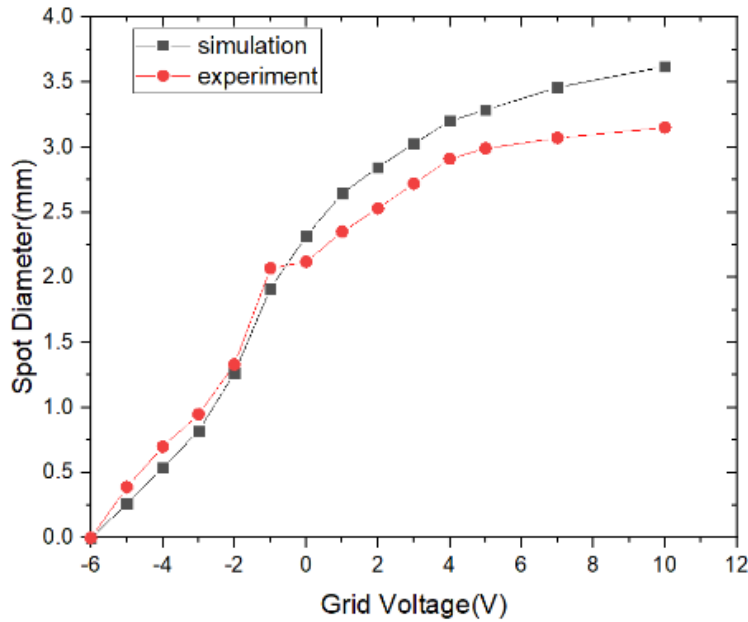

Figure 8. Effect of grid voltage on electron beam spot. (beam energy $=1000 \mathrm{eV}$, cathode current $=1.7 \mathrm{~A}$ ).

The above performance tests illustrate that the electron gun we designed has two working modes- "focus mode" and "expansion mode" although the control capability is not as good as the designed one. In the focus mode, the negative grid voltage leads to the convergence of the electron beam, the beam current decreases and the beam spot area reduces. In the expansion mode which is often happened in charge neutralization, on the contrary, the positive grid voltage expands the beam spot area and increases the beam current. 
The uniformity of the electron beam determines the effect of neutralization.The uniformity can be characterized by the results of the beam current density at all sampling points when using the pinhole scanning method. The value of beam current density is equal to the current collected by the aperture of the apertured Faraday cup divides the area of the aperture. The beam current density distribution map is generated after the density is calculated at all sampling points as shown in Figure 9a. The contour at the intersection of the purple and the black represents the outline of the beam spots. It indicates that the closer to the beam spot center, the higher the beam current density. The maximum value is $172.3 \mu \mathrm{A} / \mathrm{cm}^{2}$ in the red at the top. As can be seen in Figure $9 \mathrm{~b}$, the beam spot is roughly a Gaussian spot, from which can be inferred that the uniformity of the electron beam is good.

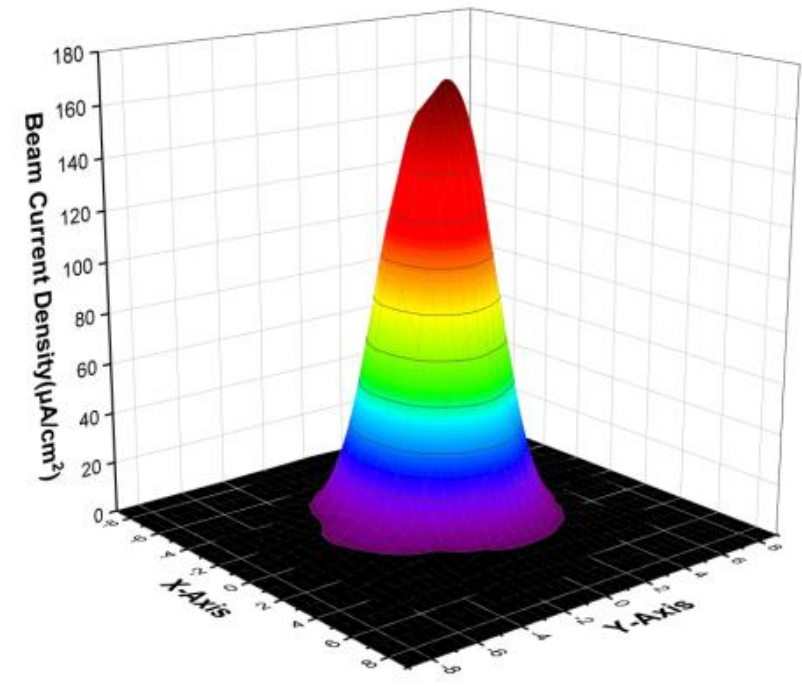

(a)

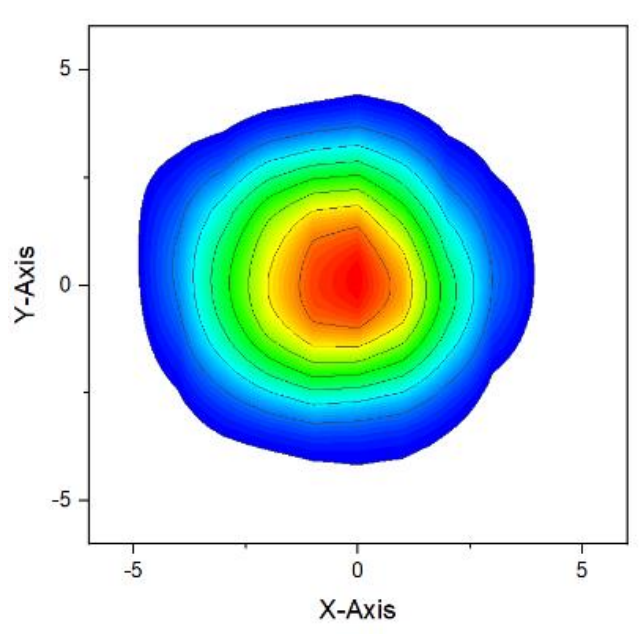

(b)

Figure 9. (a) Distribution map of beam current density; (b) the contour of beam current density.

After the beam current and beam spot are measured, the stability of the electron flood gun is further evaluated. The stability of the beam current is below $1 \%$ after warming up as shown in Figure 10 under the conditions that the acceleration voltage and cathode current are constant.

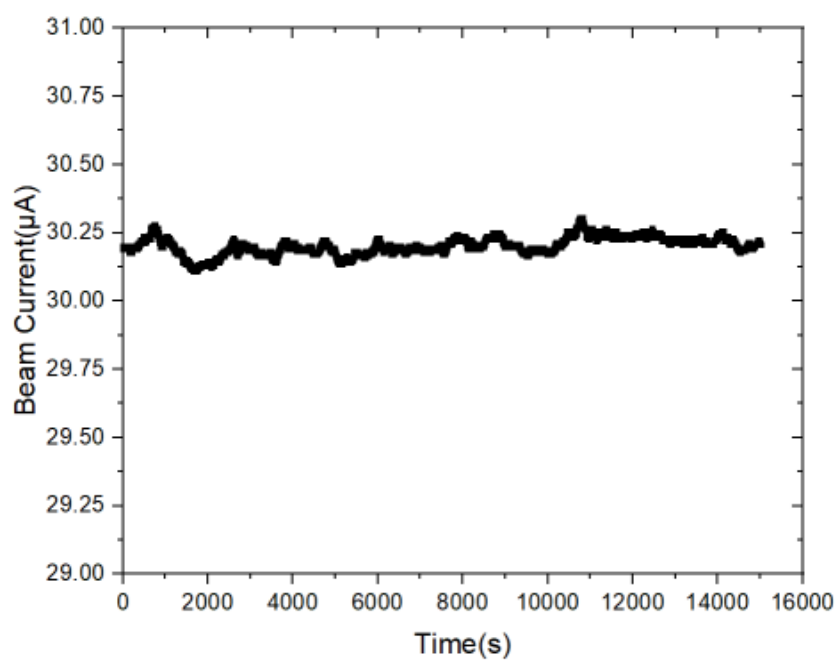

Figure 10. Stability of beam current after warming up. (beam energy $=1000 \mathrm{eV}$, cathode current $=1.7 \mathrm{~A}$ ). 


\section{Summary and Conclusions}

In this paper, we proposed a miniaturized, low-energy, large beam spot electron flood gun structure. The structural parameters of each electrode were determined by the simulation of Munro's Electron Beam Software. The electron flood gun was processed and assembled with the function of centering alignment. To measure the performances of the electron flood gun accurately and comprehensively, a new method-the pinhole scanning method-had been proposed. A test system had been built based on it. The system enables in-situ measurement of beam current and bean spot and simultaneous acquires of large amounts of data.

Experimental results and their agreement with simulation means the electron flood gun we built has the characteristic of a miniaturized, low-energy, large and uniform beam spot. These properties are sufficient to support its operation in different environments of charge neutralization. The in-depth analysis of the data of the beam performance has a guiding function to optimize the structural design and installation of the electron flood gun. We plan to integrate it into the objective lens of SEM in future work benefits from its miniaturized structure.

Author Contributions: Conceptualization, C.D. and L.H.; methodology, C.D. and L.H.; software, Y.W.; validation C.D.; formal analysis, C.D.; investigation, Y.W. and C.D.; resources, Y.W. and L.H.; data curation, C.D.; writing —original draft preparation, C.D.; writing—review and editing, C.D.; visualization, C.D.; supervision, Y.W.; project administration, L.H.; funding acquisition, L.H. All authors have read and agreed to the published version of the manuscript.

Funding: This research was funded by National Science and Technology Major Projects of China grant number (2018ZX02201).

Data Availability Statement: The data that support the findings of this study are available from the corresponding author upon reasonable request.

Acknowledgments: This work is supported by National Science and Technology Major Projects of China (2018ZX02201). The authors appreciate the useful discussions with Junbiao Liu.

Conflicts of Interest: The authors declare no conflict of interest

\section{References}

1. Cazaux, J. Mechanisms of charging in electron spectroscopy. J. Electron. Spectrosc. Relat. Phenom. 1999, 105, 155-185. [CrossRef]

2. Melchinger, A.; Hofmann, S. Dynamic double layer model: Description of time dependent charging phenomena in insulators under electron beam irradiation. J. Appl. Phys. 1995, 78, 6224-6232. [CrossRef]

3. Sessler, G. Charge dynamics in irradiated polymers. IEEE Trans. Electr. Insul. 1992, 27, 961-973. [CrossRef]

4. Cazaux, J. e-Induced secondary electron emission yield of insulators and charging effects. Nucl. Instrum. Methods Phys. Res. Sect. B Beam Interact. Mater. Atoms 2006, 244, 307-322. [CrossRef]

5. Cazaux, J. Charging in scanning electron microscopy "from inside and outside". Scanning 2004, 26, 181-203. [CrossRef] [PubMed]

6. Jbara, O.; Fakhfakh, S.; Belhaj, M.; Rondot, S. Charge Implantation Measurement on Electron-Irradiated Insulating Materials by Means of a SEM Technique. Microsc. Microanal. 2004, 10, 697-710. [CrossRef]

7. Huchital, D.A.; McKeon, R.T. Use of an Electron Flood Gun to Reduce Surface Charging in X-Ray Photoelectron Spectroscopy. Appl. Phys. Lett. 1972, 20, 158-159. [CrossRef]

8. Tomizuka, H.; Ayame, A. Charge-up Phenomena and Electron Flooding Effects on Monochromated X-Ray Photoelectron Spectroscopic Measurements of Alumina. Anal. Sci. 1994, 10, 633-639. [CrossRef]

9. Cazaux, J. About the charge compensation of insulating samples in XPS. J. Electron. Spectrosc. Relat. Phenom. 2000, 113, 15-33. [CrossRef]

10. Hofmann, S. Charging and charge compensation in AES analysis of insulators. J. Electron. Spectrosc. Relat. Phenom. 1992, 59, 15-32. [CrossRef]

11. Hunt, C.P.; Stoddart, C.T.H.; Seah, M.P. The surface analysis of insulators by SIMS: Charge neutralization and stabilization of the surface potential. Surf. Interface Anal. 1981, 3, 157-160. [CrossRef]

12. Havelund, R.; Seah, M.P.; Shard, A.G.; Gilmore, I.S. Electron Flood Gun Damage Effects in 3D Secondary Ion Mass Spectrometry Imaging of Organics. J. Am. Soc. Mass Spectrom. 2014, 25, 1565-1571. [CrossRef] [PubMed]

13. Myers, B.D.; Dravid, V.P. Variable Pressure Electron Beam Lithography (VP-eBL): A New Tool for Direct Patterning of NanometerScale Features on Substrates with Low Electrical Conductivity. Nano Lett. 2006, 6, 963-968. [CrossRef] 
14. Romig, T.; Chao, K.; Rendon, M.J.; Azrack, M. Implant charging dependence on photo resist bake and electron flood gun. In Proceedings of the International Conference on Ion Implantation Technology, Alpbach, Austria, 17-22 September 2000; pp. 558-560.

15. Dresner, J.; Goldstein, B. Dissociation of $\mathrm{MgO}$ films under heat and electron bombardment and its effects on secondary emission. J. Appl. Phys. 1976, 47, 1038-1043. [CrossRef]

16. Jbara, O.; Fakhfakh, S.; Belhaj, M.; Cazaux, J.; Rau, E.; Filippov, M.; Andrianov, M. A new experimental approach for characterizing the internal trapped charge and electric field build up in ground-coated insulators during their e- irradiation. Nucl. Instrum. Methods Phys. Res. Sect. B Beam Interact. Mater. Atoms 2002, 194, 302-310. [CrossRef]

17. Toth, M.; Thiel, B.; Donald, A. Interpretation of secondary electron images obtained using a low vacuum SEM. Ultramicroscopy 2003, 94, 71-87. [CrossRef]

18. Gleizer, J.Z.; Vekselman, V.; Yatom, S.; Felsteiner, J.; Krasik, Y.E. Low-energy electron beam source. Radiat. Eff. Defects Solids 2011, 166, 389-398. [CrossRef]

19. Erdman, P.W.; Zipf, E.C. Low-voltage, high-current electron gun. Rev. Sci. Instrum. 1982, 53, 225-227. [CrossRef]

20. Cantero, E.; Sosa, A.; Andreazza, W.; Bravin, E.; Lanaia, D.; Voulot, D.; Welsch, C. Design of a compact Faraday cup for low energy, low intensity ion beams. Nucl. Instrum. Methods Phys. Res. Sect. A Accel. Spectrometers Detect. Assoc. Equip. 2016, 807, 86-93. [CrossRef]

21. Elleaume, P.; Fortgang, C.; Penel, C.; Tarazona, E. Measuring Beam Sizes and Ultra-Small Electron Emittances Using an X-ray Pinhole Camera. J. Synchrotron Radiat. 1995, 2, 209-214. [CrossRef]

22. Chisholm, T. Spot-size measurement in an electron-beam pattern generator. J. Vac. Sci. Technol. B Microelectron. Process. Phenom. 1988, 6, 2066. [CrossRef] 